\title{
A $0535+26$ in outburst: magnetospheric instabilities and accretion geometry
}

\section{Caballero ${ }^{* a}$, U. Kraus ${ }^{a}$, K. Postnov ${ }^{b}$, A. Santangelo ${ }^{a}$, P. Kretschmar ${ }^{c}$, D. Klochkov ${ }^{a}$ and R. Staubert ${ }^{a}$}

${ }^{a}$ Institut für Astronomie und Astrophysik, University of Tübingen

Sand 1, 72076 Tübingen, Germany

${ }^{b}$ Sternberg Astronomical Institute, Moscow University

119992 Moscow, Russia

${ }^{c}$ ISOC, European Space Astronomy Centre, European Space Agency

aaPO Box 78, 28691 Villanueva de la Cañada, Madrid, Spain

E-mail:

isabel.caballero@astro.uni-tuebingen.de, krauseastro.uni-tuebingen.de,

kpostnov@gmail.com, santangelodastro.uni-tuebingen.de,

Peter.Kretschmar@esa.int, klochkov@astro.uni-tuebingen.de,

stauberteastro.uni-tuebingen.de

The Be/X-ray binary A 0535+26 showed a normal (type I) outburst in August/September 2005, which reached a maximum X-ray flux of $400 \mathrm{mCrab}$ in the $5-100 \mathrm{keV}$ range. The outburst was observed by INTEGRAL and RXTE.

The energy of the fundamental cyclotron line has been measured with INTEGRAL and RXTE at $\sim 45 \mathrm{keV}$. Flaring activity was observed during the rise to the peak of the outburst. RXTE observations during one of these flares found the energy of the fundamental cyclotron line shifted to a significantly higher position than during the rest of the outburst, where it remains constant. Also, the energy-dependent pulse profiles during the flare differ significantly from the rest of the outburst. These differences have been interpreted with the presence of magnetospheric instabilities at the onset of the accretion.

A decomposition method is applied to A $0535+26$ pulse profiles. Basic assumptions of the method are that the asymmetry observed in the pulse profiles is caused by non-antipodal magnetic poles, and that the emission regions have axisymmetric beam patterns. Using pulse profiles obtained from RXTE observations, the contribution of the two emission regions has been disentangled. Constraints on geometry of the pulsar and a possible solution of the beam pattern are given. First results of the comparison of the reconstructed beam pattern with a geometrical model that includes relativistic light deflection are presented.

7th INTEGRAL Workshop

September 8-112008

Copenhagen, Denmark

${ }^{*}$ Speaker. 


\section{Introduction}

The Be/X-ray binary A $0535+26$ was discovered in 1975 by [1]. It is a transient source, in an eccentric orbit $(e=0.47)$ of $\sim 110.3$ days orbital period [2]. Since its discovery it has shown 5 giant (type II) outbursts in 1980 [3], June 1982 [4], March 1989 [5], February 1994 [2] and May 2005 [6]. The last one in 2005 could not be observed in X-rays by most astronomical instruments due to Sun constraints. A subsequent normal (type I) outburst took place in August/September 2005 [7] associated with the periastron passage. The outburst led to our INTEGRAL and RXTE observations.

The source spectrum presents cyclotron resonance scattering features at $\sim 45 \mathrm{keV}$ and $\sim 100 \mathrm{keV}$ discovered by HEXE [8]. The INTEGRAL and RXTE observations have provided the first high accuracy measurements of the cyclotron line energy of A 0535+26. RXTE monitoring has allowed to study the evolution of the cyclotron line energy at different luminosities, and like in other sources ([9], [10], [11]) it was found to vary with the X-ray luminosity [12].

Flaring activity has been observed during the rise to the peak of the outburst. RXTE observations from one of these flares have revealed a different spectral and timing behavior of the source. These results have been presented in [12], and a possible interpretation of the observed changes is proposed in [13]. A review of the observations and interpretation is given in Sec. 2. In Sec. 3 results from a decomposition analysis of A $0535+26$ energy-dependent pulse profiles are shown. The method and assumptions are described in Sec.3.1. A more detailed description is given in [14], and therefore all the formal derivations and technical details are not repeated here. We find a possible decomposition of the pulse profiles, that has allowed to reconstruct the visible part of the beam pattern of A $0535+26$, and to extract information on the geometry of the system (Sec. 3.2). The reconstructed beam pattern is used as input for a geometrical model that takes into account general relativity effects. Preliminary results are presented in Sec.3.2.3. Sec. 4 contains a summary and conclusions.

\section{Magnetospheric instabilities}

Flaring activity has been detected during the rise to the peak of the 2005 outburst. RXTE ASM and Swift BAT light curves are shown in Fig. 1. INTEGRAL performed a $\sim 200$ ks observation near the peak of the outburst, and RXTE monitored the complete outburst with an exposure time of $\sim 140 \mathrm{ks}$, including three observations of one flare during the rise to the peak. The INTEGRAL and RXTE observations are indicated in Fig. 1.

The pulse period of the pulsar has been studied in detail during the outburst. A constant spin period (within uncertainties) is measured during first part of the outburst, $P=103.3960(5) \mathrm{s}$, followed by a spin-up of $\dot{P}=(-1.69 \pm 0.04) \times 10^{-8} \mathrm{ss}^{-1}$ (measured at MJD 53618) at periastron. For the first time during a normal outburst a spin-up is measured for A $0535+26$, providing evidence for the presence of an accretion disk [12].

Energy-dependent pulse profiles during the pre-outburst flare differ considerably from those obtained during the main part of the outburst, as shown in Fig. 2 (left). During the main part of the outburst, a strong change of the pulse profiles above the cyclotron energy takes place. However, during the flare there is a smooth evolution of the pulse profiles above the cyclotron energy. The 


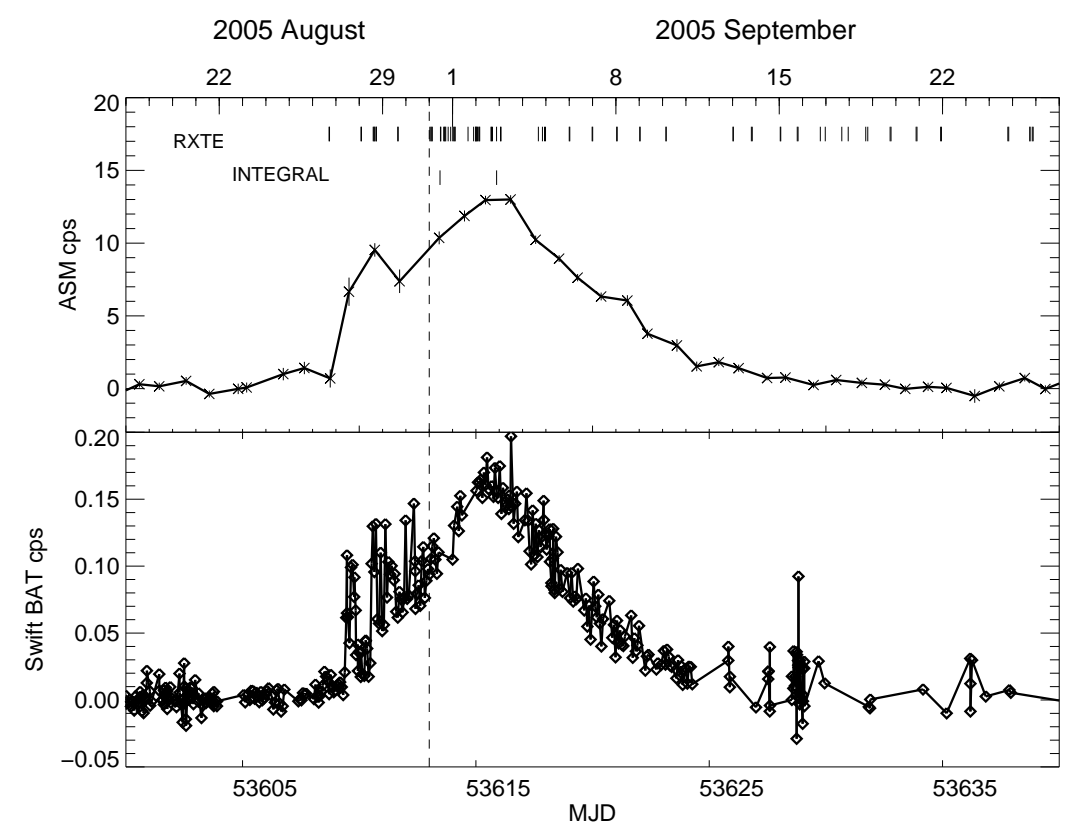

Figure 1: A 0535+26 RXTE ASM and Swift BAT light curves during the normal (type I) outburst in August/September 2005. The dashed vertical line indicates the periastron time. The RXTE and INTEGRAL observations are indicated in the upper panel.

drastic change above the cyclotron energy during the main peak has been interpreted in [13] as a weakening of the fan-like component of the beam due to the energy dependence of the crosssection for ordinary photons. The cyclotron line energy remains constant during the main part of the outburst. Near the peak, INTEGRAL measured $E_{\mathrm{cyc}}=45.9 \pm 0.3 \mathrm{keV}$ [15]. A significant change in the cyclotron line is measured during the pre-outburst flare, reaching $E_{\mathrm{cyc}}=52.0_{-1.4}^{+1.6} \mathrm{keV}$. The cyclotron line evolution during the outburst is shown in Fig. 2 (right).

The flaring activity observed has been explained as due to low-mode magnetospheric instability that develops at the onset of the accretion, in the thin boundary layer between the accretion disk and neutron star magnetosphere. The matter accumulated in the boundary layer rapidly falls onto the neutron star surface close to the magnetic poles, along different magnetic field lines by which stationary accretion proceeds. This explains the differences in the pulse profiles and the change in the cyclotron energy during the flare compared to the rest of the outburst. Details are given in [13]. The present analysis will be performed (and is applicable) only to the stable pulse profiles in the main outburst.

\section{Pulse profile decomposition}

Geometrical models of filled and hollow accretion columns of accreting neutron stars, including relativistic light deflection, have been computed in [16] and [17]. These models give the beam pattern or energy-dependent flux of one emission region as a function of the angle, as seen by a distant observer. Introducing the rotation of the pulsar and its geometry, i.e., the orientation of the rotation axis with respect to the direction of observation and the location of the two poles, the 

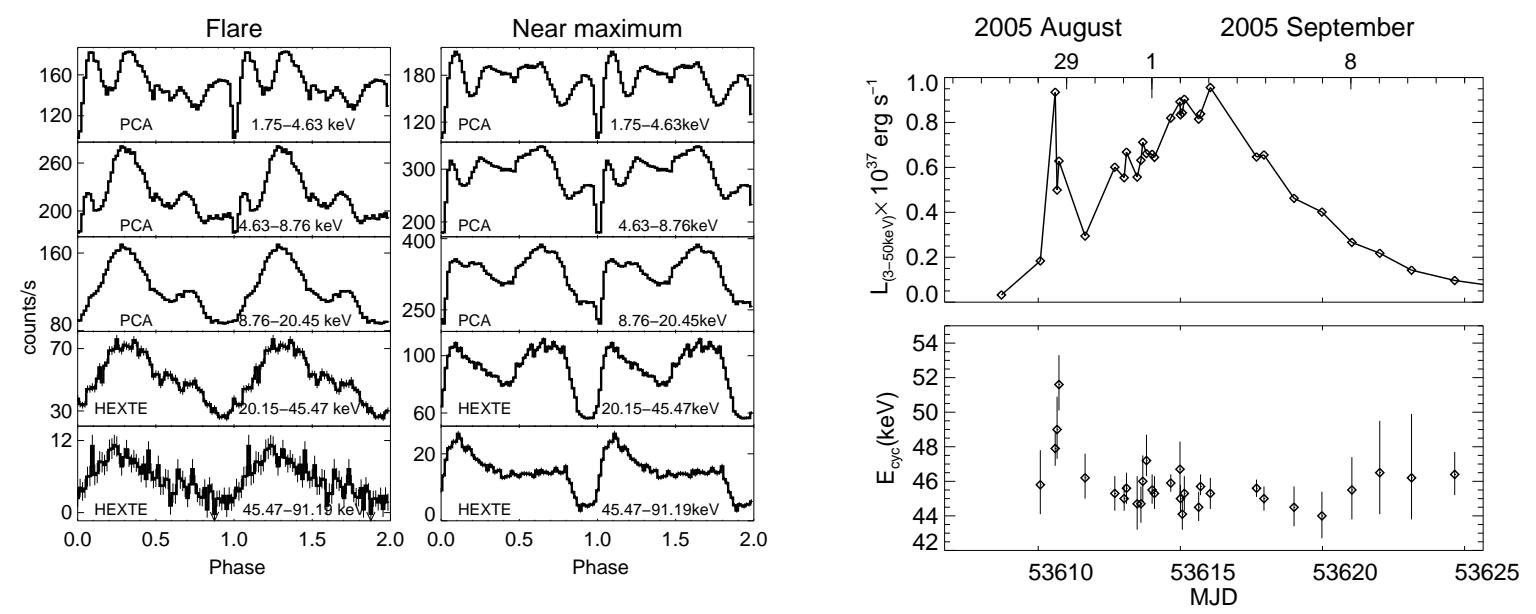

Figure 2: Left: A $0535+26$ energy-dependent pulse profiles during the flare and near the peak of the August/September 2005 normal (type I) outburst. Right: PCA light curve of the outburst (top) and evolution of the cyclotron line energy (bottom).

pulsed emission from each of the two poles or single-pole pulse profiles that a distant observer would see can be modeled. The sum of the single-pole contributions gives the total pulse profile.

In this work we apply a decomposition method that inverts the process described above. From the observed pulse profile the single-pole pulse profiles are obtained, and from the single-pole contributions information on the geometry of the neutron star and the beam pattern is extracted. This method has been applied to the accreting pulsars Cen X-3 [18] and Her X-1 [19]. Of course the backwards process is not straightforward and involves ambiguities which will be discussed.

\subsection{Method}

\subsubsection{Assumptions}

The basic assumption, which is often adopted in model calculations, is that the emission regions at the magnetic poles are axisymmetric. The beam pattern from one pole is then only a function of the angle $\theta$ between the direction of observation and the magnetic axis. This makes the single-pole pulse profile necessarily symmetric. One of the symmetry points will be the instant when the magnetic axis is closest to the line of sight, and the other symmetry point will be half a period later, with the magnetic axis pointing away from the observer.

Assuming an ideal dipole magnetic field, the above assumption necessarily implies a symmetric total pulse profile. It can be shown that a small displacement of one of the magnetic poles from the antipodal position can explain the asymmetry in the sum of the single-pole contributions. Therefore the assumption of an ideal dipole field is modified, introducing a small offset from an ideal dipole field.

Another assumption made is that the two emission regions are the same, i.e., have the same beam pattern. This implies that each of the two poles will make visible one section of the same beam pattern. Depending on the geometry of the neutron star and the angle of observation, those two sections will in some cases have coincident parts. This assumption has been tested with the 
accreting pulsars Cen X-3 [18] and Her X-1 [19]. In those cases an overlapping region was found, in agreement with the assumption of two equal emission regions.

\subsubsection{Decomposition into single-pole pulse profiles}

The first step of the analysis is to express the original pulse profile as a Fourier series. The total pulse profile $\mathrm{F}$ is written as:

$$
F(\Phi)=\frac{1}{2} u_{0}+\sum_{k=1}^{n / 2-1}\left[u_{k} \cos (k \Phi)+v_{k} \sin (k \Phi)\right]+u_{n / 2} \cos \left(\frac{n}{2} \Phi\right)
$$

where $\mathrm{n}$ is the number of bins of the original pulse profile and $\Phi$ is the phase. Eq. 3.1 gives a valid representation of the original pulse profile at all phases if the Fourier transform of $\mathrm{F}$ approaches to zero as the frequency approaches $n / 2$. If this is not the case (phenomenon called aliasing), less Fourier coefficients are taken into account to describe the original pulse profile.

The single-pole pulse profiles $f_{1}$ and $f_{2}$ are described by the following symmetric functions:

$$
\begin{gathered}
f_{1}(\Phi)=\frac{1}{2} c_{0}+\sum_{k=1}^{n / 2} c_{k} \cos \left[k\left(\Phi-\Phi_{1}\right)\right] \\
f_{2}(\Phi)=\frac{1}{2} d_{0}+\sum_{k=1}^{n / 2} d_{k} \cos \left\{k\left[\Phi-\left(\Phi_{2}+\pi\right)\right]\right\}
\end{gathered}
$$

$\Phi_{1}$ and $\Phi_{2}$ are the symmetry points of $f_{1}(\Phi)$ and $f_{2}(\Phi)$ respectively. Formally, a decomposition of $\mathrm{F}$ into two symmetric functions exists for every choice of their symmetry points $\Phi_{1}$ and $\Phi_{2}$. For convenience, we use the parameter $\Delta:=\pi-\left(\Phi_{1}-\Phi_{2}\right)$, that represents the azimuthal displacement of one pole with respect to the antipodal position (see Sec. 3.1.3). All formal decompositions will be contained in the parameter space $\Phi_{1}-\Delta$, with $0 \leq \Phi_{1} \leq \pi$ and $0 \leq \Delta \leq \pi / 2$. Once the formal decompositions are calculated, physical criteria are applied to decrease the number of decompositions to physically meaningful ones. Those criteria are the following:

- "positive criterion": both $f_{1}(\Phi)$ and $f_{2}(\Phi)$ must be positive, since they represent photon fluxes.

- "non-ripples criterion": the single-pole contributions $f_{1}(\Phi)$ and $f_{2}(\Phi)$ should not be much more complicated than the original pulse profile. Individual pulse profiles with many peaks that cancel out in the sum are not accepted.

- the same symmetry points must give valid decompositions in all energy bands.

Once a possible decomposition is found, the symmetry points for each of the two poles $\Phi_{1}$ and $\Phi_{2}$, and the parameter $\Delta$, related to the position of the emission regions on the neutron star, are determined. 


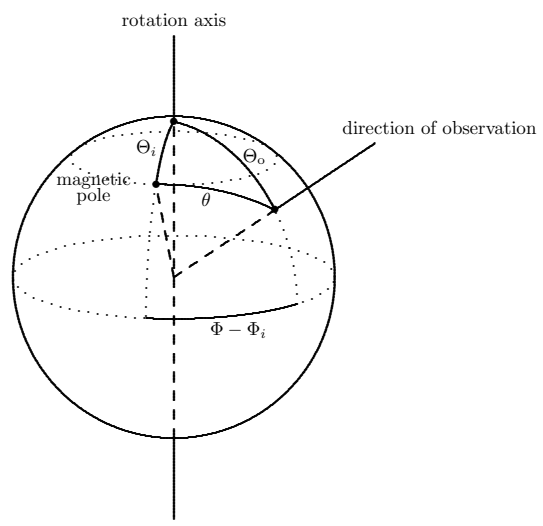

Figure 3: Schematic view of a rotating neutron star. A spherical coordinate system is used, with the rotation axis as polar axis. $\Theta_{i}$ is the polar angle of the $i$ th pole. $\Theta_{0}$ is the polar angle of the direction of observation. The angle $\theta$ between the magnetic pole and the direction of observation changes with the rotation angle $\Phi$. Figure from [14].

\subsubsection{From single-pole pulse profiles to beam pattern}

In Fig. 3 a schematic view of a rotating neutron star is shown. A spherical coordinate system is used with the rotation axis as polar axis. As was explained above, the beam pattern is assumed to be axisymmetric, and therefore to depend only on the angle between the direction of observation and the magnetic axis $\theta$. The value of $\theta$ changes with the rotation angle $\Phi$. Depending on the position of the poles with respect to the rotation axis and depending on the direction of observation with respect to the magnetic axis, we will only observe a section of the beam pattern for each pole.

Applying the cosine formula to the spherical triangle in Fig. 3, we obtain $\theta$ as a function of the phase $\Phi$ :

$$
\cos \theta=\cos \Theta_{0} \cos \Theta_{i}+\sin \Theta_{0} \sin \Theta_{i} \cos \left(\Phi-\Phi_{i}\right)
$$

where $\Theta_{0}$ is the polar angle of the direction of observation, $\Theta_{i}$ the polar angle of the $i$ th pole, and $\Phi_{i}$ one symmetry point for the ith pole.

The intrinsic pulsar geometry is shown in Fig. 4. A complete description of the pulsar can be given in terms of the polar angles $\Theta_{1}$ and $\Theta_{2}$, and the difference in their azimuthal angles $\Phi_{1}-\Phi_{2}=\pi-\Delta$. The angular distance $\delta$ between the location of the second magnetic pole and the point that is antipodal to the first magnetic pole can be used as a measure for the deviation from an ideal dipole field. From Fig. 4:

$$
\cos \delta=-\cos \Theta_{2} \cos \Theta_{1}+\sin \Theta_{2} \sin \Theta_{1} \cos \Delta
$$

Considering the beam pattern as a function of $\cos \theta$ and the single pole pulse profiles as functions of $\cos \left(\Phi-\Phi_{i}\right)$, as the relation between $\cos \theta$ and $\cos \left(\Phi-\Phi_{i}\right)$ is linear (Eq.3.4), there is no distortion between the two functions. Therefore, once we have the single-pole pulse profiles, plotting them as a function of $\cos \left(\Phi-\Phi_{i}\right)$ we will obtain two sections of the beam pattern. In some cases an overlapping region will emerge. Using geometrical properties, it can be shown that 


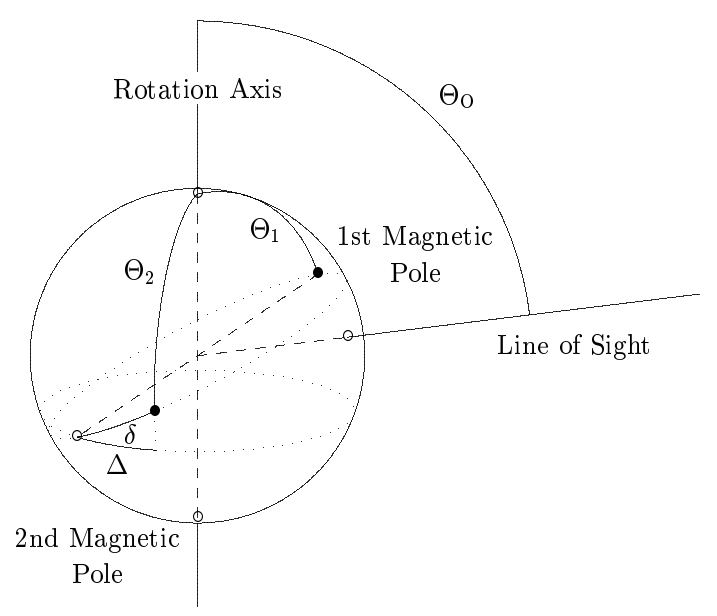

Figure 4: Intrinsic geometry of the pulsar. With the rotation axis as polar axis, the magnetic poles are located at polar angles $\Theta_{1}$ and $\Theta_{2}$. The angular distance $\delta$ between the second magnetic pole and the point that is antipodal to the first magnetic pole gives the deviation from an ideal dipole field.Figure from [19].

the relative position of the two single-pole profiles (in terms of the total pulse profile) are related to the position of the two poles. This requires an independent determination of the direction of observation. Details can be found in [14].

\subsection{Application to A $0535+26$}

RXTE HEXTE energy-dependent pulse profiles of A 0535+26 during the August/September 2005 normal outburst have been analyzed. Pulse profiles obtained during the main part of the outburst have been selected for the analysis, as those profiles appear to be very stable, not only during the outburst but also compared to historical observations. Several observations have been used, for a total exposure time of $\sim 20 \mathrm{ks}$.

\subsubsection{Search for acceptable decompositions}

The energy-dependent pulse profiles are written as Fourier series. These functions are then written as the sum of two symmetric functions $f_{1}(\Phi)$ and $f_{2}(\Phi)$. To search for physically meaningful decompositions, the $\Phi_{1}-\Delta$ parameter space is divided in $1^{\circ} \times 1^{\circ}$ boxes. All the formal decompositions are represented in this plane. We apply the "non-negative criterion". Out of all the decompositions, only those for which $f_{1}(\Phi)$ and $f_{2}(\Phi)$ are positive are accepted. Fig. 5 (left) shows where in the $\Phi_{1}-\Delta$ plane positive decompositions have been found. The "non-ripples criterion" is then applied. A quality function is defined, which counts the number of peaks in one profile. To avoid having to study all the decompositions, similar ones are grouped into types. We study the representative of each type. Fig. 5 (right) shows where the highest ranked profile representatives were found. We have examined all these decompositions in different energy ranges, dividing the parameter space in five regions $A, B, C, D$ and $E$.

Combining the results of the analysis in different energy ranges and for different observations, we find a best decomposition of the original pulse profiles in region A. They are shown in Fig. 6. The dotted horizontal line corresponds to the remaining unmodulated flux available to distribute 

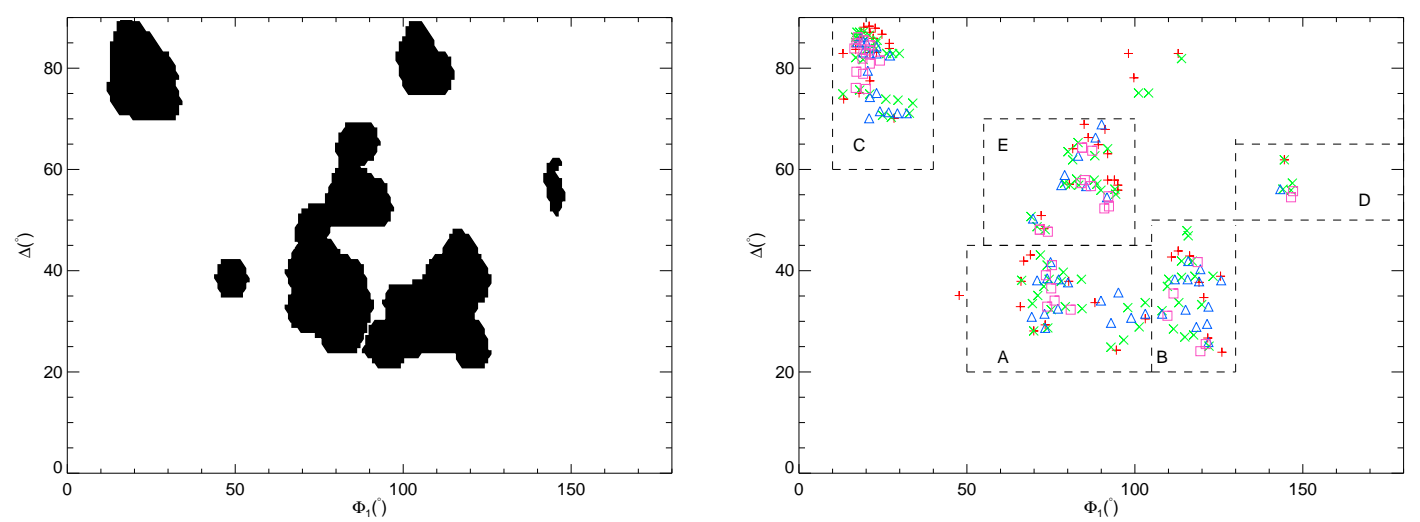

Figure 5: Left: Result of applying the "non-negative" criterion: decompositions outside the black area are discarded. Right: Highest ranked decompositions grouped in five regions $A, B, C, D$ and $E$. The different symbols represent different energy ranges: +: (18.3-30.9) keV, $\times$ : (30.9-44.5) keV , $\triangle$ : (44.5-59.1) keV, $\square:(59.1-99.8) \mathrm{keV}$

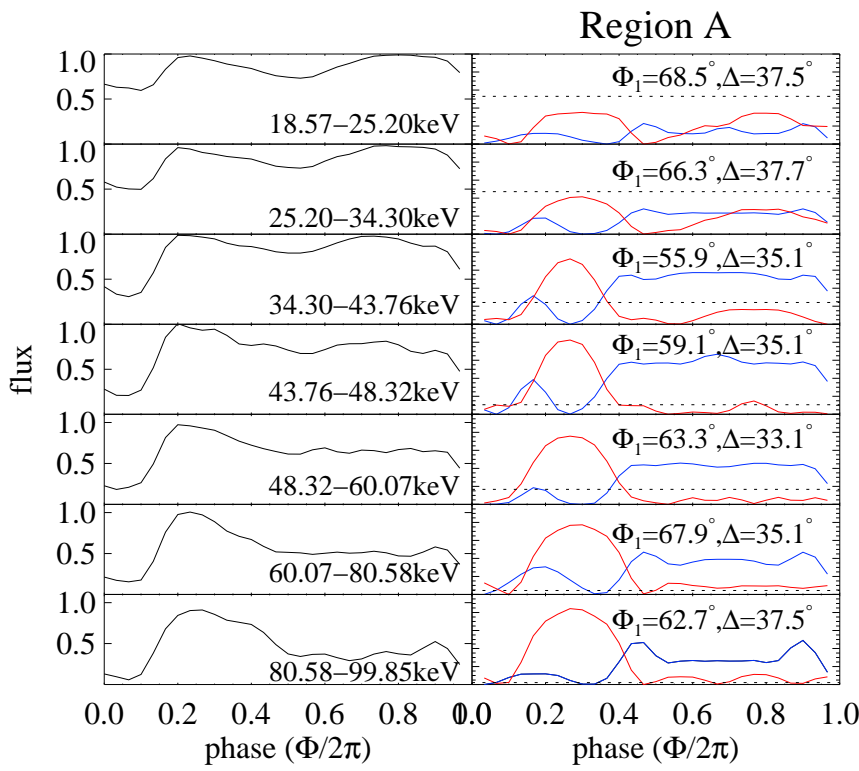

Figure 6: Best decomposition of the original pulse profiles (left) in two symmetric functions (right).

between the two symmetric functions that can not be determined from the decomposition. The minima of the two symmetric functions have been shifted to zero, so that the sum of the two symmetric functions plus the unmodulated flux reproduces the original pulse profile. The values of $\Phi_{1}, \Delta$ and $\Phi_{2}$ (using $\Delta=\pi-\left(\Phi_{1}-\Phi_{2}\right)$ ) can not be more accurate than the $12^{\circ}$ bin resolution, and we therefore use this value as uncertainty for the symmetry points. Average values for the best decomposition in region $A$ are $\Phi_{1}=73^{\circ} \pm 12^{\circ}, \Delta=33^{\circ} \pm 12^{\circ}$ and $\Phi_{2}+\pi=106^{\circ} \pm 12^{\circ}$

Decompositions in regions $C$ and $E$ are discarded because they present a very strong anticorrelation in the main peaks which seems artificial, not expected from two independent emission regions. Decompositions in regions $B$ and $D$ have also been discarded, because the single-pole 

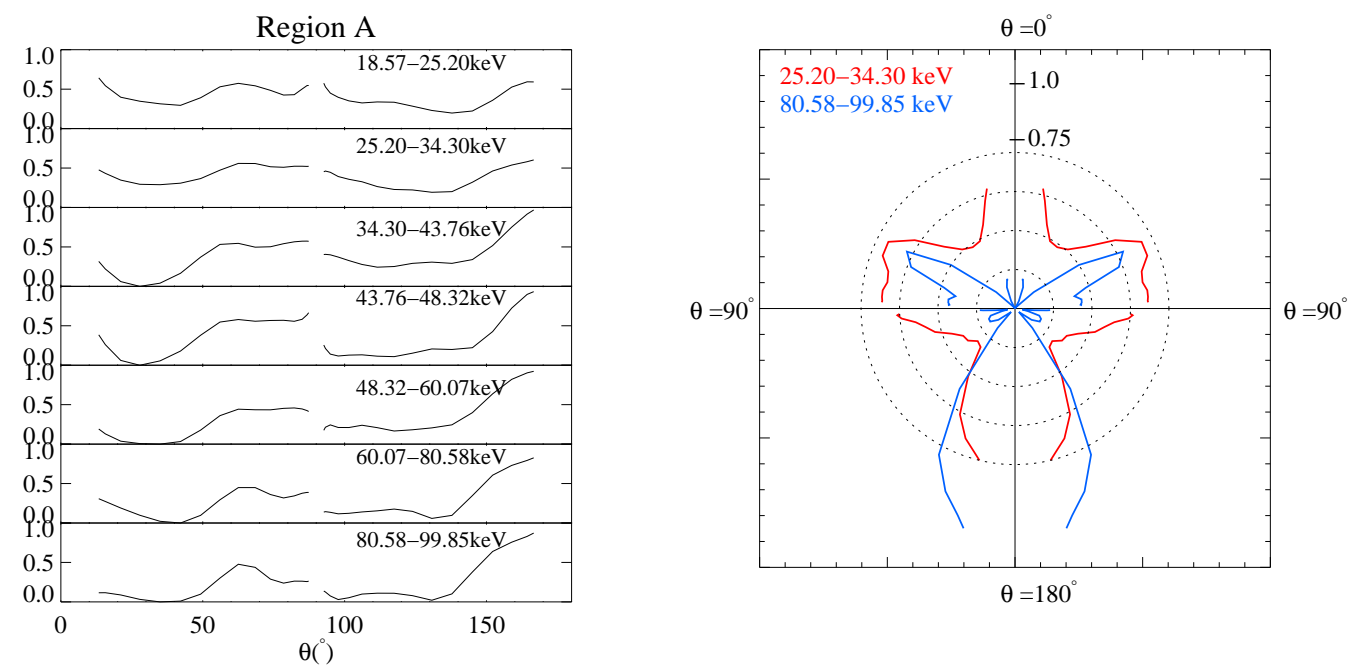

Figure 7: Reconstructed beam pattern of A $0535+26$ in different energy ranges. Left: linear representation. Right: polar diagram.

pulse profiles present an anti-correlation in many small features that cancel out in the sum, also not expected from two independent emission regions. Another argument to reject decompositions in regions $C, D$ and $E$ is that they all present higher values of $\Delta$. Under the assumption of slightly displaced magnetic poles, smaller values of $\Delta$ are more likely to be real. This was the case in the analysis of the accreting pulsars Cen X-3 [18] and Her X-1 [19], where the best decompositions were found for small values of $\Delta$. A further argument against decompositions in regions $B, C$ and $D$ emerges in the reconstruction of the beam pattern from the single-pole contributions (see Sec. 3.2.2).

\subsubsection{From single-pole pulse profiles to geometry and beam pattern}

Doing the proper transformation of the single pole pulse profiles into undistorted sections of the beam pattern, an overlapping region does not emerge. However, under the assumption of two identical emission regions, the two sections can almost be connected to each other, with a small gap in between. This was not possible for the decompositions in regions $B, C$ and $D$. The shift between the two sections is related to the position of the magnetic poles and the direction of observation (see [14] for details). The inclination of the system is $i=37 \pm 2^{\circ}$ [20]. Assuming that the rotation axis of the neutron star is perpendicular to the orbital plane, $i=\Theta_{0}$. We can therefore obtain the location of the poles $\Theta_{1}$ and $\Theta_{2}$. The angular distance between the location of the second pole and the point that is antipodal to the first pole $\delta$ can be estimated using Eq. 3.5. The estimated values for the position of the magnetic poles and the offset are $\Theta_{1} \approx 50^{\circ}, \Theta_{2} \approx 130^{\circ}, \delta \approx 25^{\circ}$. It is then possible to plot the reconstructed sections of the beam pattern as a function of $\theta$. The two sections of the beam pattern are reconstructed for $\theta \in\left(13^{\circ}-87^{\circ}\right)$ and $\theta \in\left(93^{\circ}-167^{\circ}\right)$. The reconstructed beam pattern is shown in Fig. 7.

\subsubsection{Interpretation of beam pattern}

A characteristic feature of the reconstructed beam pattern is a minimum observed in the flux 


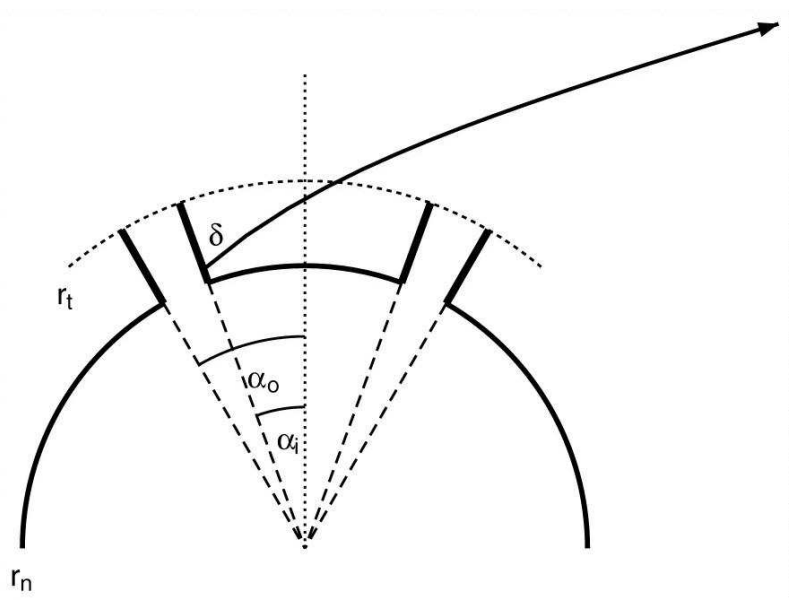

Figure 8: Geometrical model of hollow column. Figure from [16]

between $\theta \approx 30^{\circ}-40^{\circ}$. This feature is present at all energies and we therefore believe that it could be related to the geometry of the accretion. (It is also present at lower energies, seen from the analysis of PCA data [23]). Filled column models give a beam pattern in which the flux decreases at low values of $\theta$, corresponding to the instant when the observer looks along the accretion stream [17]. Introducing a hollow column plus a halo created on the neutron star surface around the column walls from scattered radiation emitted from the walls would explain the flux at low values of $\theta$ and the minimum as $\theta$ increases when the observer looks directly into the column.

The steep increase in flux at high values of theta $\left(\theta>120^{\circ}\right)$ could be due to gravitational light bending, which produces a similar feature in model calculations.

To obtain estimates on the size of the accretion column, a model for a hollow column plus a halo has been calculated for $\theta \in\left(0^{\circ}, 40^{\circ}\right)$. Geometrical models of filled columns, including the formation of a halo around the accretion column, were presented in [17], where the relative importance of the different components (halo-column) to the observed flux was studied. In the work presented here, the modelling is performed as in [17], but introducing a hollow column. A detailed study of this model will be presented elsewhere. Beam patterns are computed using raytracing [21] and include relativistic light deflection [22].

The emission of the column wall is assumed to be a blackbody of temperature $T_{e f f}$, assumed to be isotropic. Part of the radiation that leaves the column hits the neutron star surface, creating a luminous halo around the column walls. This emission is assumed to be thermal. Schwarzschild metric is used to calculate photon paths. This is appropriate for A $0535+26$ because it is a slowly rotating neutron star $\left(P_{s p i n} \sim 103.4 s\right)$. The Schwarzschild radius is $r_{S}=2 G M / c^{2}$ and the neutron star radius $r_{n}$. The accretion funnel has an inner half-opening angle $\alpha_{i}$ and outer half-opening angle $\alpha_{0}$. A radiative shock is assumed to form close to the neutron star surface at radial coordinate $r_{t}$. See Fig. 8 for a sketch of the geometry of the hollow column. Below the shock, the column is optically thick and radiation is emitted from the inner and outer walls. Above the shock, the accretion is assumed to be in free-fall.

The mass and radius of the neutron star are taken to be $M_{n}=1.4 \mathrm{M}_{\odot}$ and $r_{n}=10 \mathrm{~km}$. The radiative shock is formed at $r_{t}=10.5 \mathrm{~km}$, and an asymptotic luminosity per pole of $L_{\infty}=0.8 \times$ 

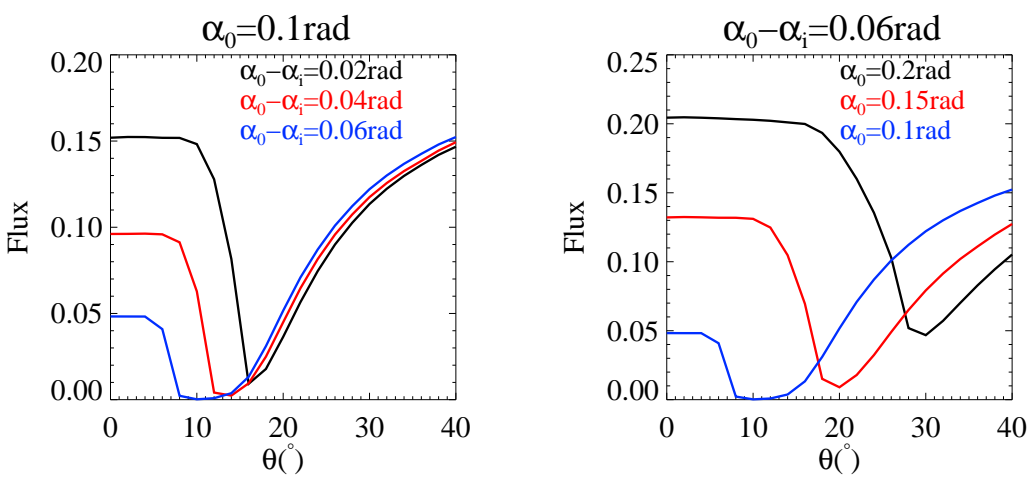

Figure 9: Beam pattern models for hollow column with halo for different column thickness and the same opening angle (left) and different opening angles with same column thickness (right), calculated for $\theta \in$ $\left(0^{\circ}, 40^{\circ}\right)$.

$10^{37} \mathrm{ergs}^{-1}$ is taken. The cyclotron energy, used to calculate the magnetic scattering cross-section, is set to $E_{\mathrm{cyc}}=45 \mathrm{keV}$ at the magnetic pole. Beam patterns have been computed for $7.6 \mathrm{keV}$ photons.

Different models have been computed for different values of the inner and outer half-opening angles. For each model, the temperature of the Plank spectrum emitted by the column wall and the density that the accreting material has at the base of the free-fall section follow from the assumed geometry and the value of $L_{\infty}$. The parameters used for each model are listed in Table 1. Results from the model calculations are shown in Fig. 9.

Table 1: Model parameters used in the computation of beam patterns for A $0535+26$, for $\theta \in\left(0^{\circ}, 40^{\circ}\right)$.

\begin{tabular}{ccccc}
\hline model & $\alpha_{i}(\mathrm{rad})$ & $\alpha_{0}(\mathrm{rad})$ & $k T(\mathrm{keV})$ & $\rho\left(10^{-5} \mathrm{~g} / \mathrm{cm}^{-3}\right)$ \\
\hline 1 & 0.08 & 0.1 & 4.1 & 16 \\
2 & 0.06 & 0.1 & 4.1 & 9 \\
3 & 0.04 & 0.1 & 4.1 & 6.8 \\
4 & 0.09 & 0.15 & 3.7 & 4 \\
5 & 0.14 & 0.2 & 3.5 & 2.8 \\
\hline
\end{tabular}

By comparing the A $0535+26$ reconstructed beam pattern (Fig. 7) with the models in Fig. 9, it can be seen that the shape of the models reproduce well the shape of the reconstructed beam pattern. We can estimate the half-opening angle and column thickness to be $\alpha_{0}=0.2 \mathrm{rad} \sim 11.5^{\circ}$ and $\alpha_{0}-\alpha_{i}=0.06 \mathrm{rad} \sim 3.4^{\circ}$. Those are preliminary results from an ongoing work that will be presented in [23].

\section{Summary and conclusions}

In this work results from spectral and timing analysis of A $0535+26$ during a flare that took place in the rise to the peak of the August/September 2005 normal outburst compared to the rest of the outburst have been summarized. The differences observed in the pulse profiles and in the cyclotron energy can be explained with magnetospheric instabilities that develop in the onset of the accretion. Those results and interpretation can be found in [12] and [13] respectively. 
In the second part of the work, a decomposition analysis has been applied to the A $0535+26$ energy-dependent pulse profiles. A dipole magnetic field is assumed with axisymmetric emission regions. The asymmetry in the total pulse profiles is explained with a small offset from one of the magnetic poles from the antipodal position. We find a physically acceptable decomposition of the pulse profiles that allows to extract information on the geometry of the pulsar. We obtain $\Theta_{1} \approx 50^{\circ}$ and $\Theta_{2} \approx 130^{\circ}$ for the position of the magnetic poles, and an offset of $\delta \approx 25^{\circ}$. The visible section of the beam pattern has been reconstructed. First results from a geometrical model of hollow column that includes the formation of a halo are used to interpret the main features of the reconstructed beam pattern of A $0535+26$. Further details will be presented in [23].

\section{References}

[1] Rosenberg, F. D., Eyles, C. J., Skinner, G. K., \& Willmore, A. P. 1975, Nature, 256, 628

[2] Finger, M. H., Wilson, R. B., \& Harmon, B. A. 1996, ApJ, 459, 288

[3] Nagase, F., Hayakawa, S., Kunieda, H., et al. 1982, ApJ, 263, 814

[4] Sembay, S., Schwartz, R. A., Orwig, L. E., Dennis, B. R., \& Davies,S. R. 1990, ApJ, 351, 675

[5] Makino, F., Cook, W., Grunsfeld, J., et al. 1989, IAU circ, 4769

[6] Tueller, J., Ajello, M., Barthelmy, S., et al. 2005, ATel, 504

[7] Finger, M. H. 2005, ATel, 676

[8] Kendziorra, E., Kretschmar, P., Pan, H. C., et al. 1994, A\&A, 291, L31

[9] Mowlavi, N., Kreykenbohm, I., Shaw, S. E., et al. 2006, A\&A, 451, 187

[10] Tsygankov, S. S., Lutovinov, A. A., Churazov, E. M., \& Sunyaev, R. A. 2006, MNRAS, 371, 19

[11] Staubert, R., Shakura, N. I., Postnov, K., et al. 2007, A\&A, 465, L25

[12] Caballero, I., Santangelo, A., Kretschmar, P., et al. 2008, A\&A, 480, L17

[13] Postnov, K., Staubert, R., Santangelo, A., et al. 2008, A\&A, 480, L21

[14] Kraus, U., Nollert, H.-P., Ruder, H., \& Riffert, H. 1995, ApJ, 450,763

[15] Caballero, I., Kretschmar, P., Santangelo, A., et al. 2007, A\&A , 465,L21

[16] Kraus, U. 2001, ApJ, 563, 289

[17] Kraus, U., Zahn, C., Weth, C., \& Ruder, H. 2003, ApJ, 590, 424

[18] Kraus, U., Blum, S., Schulte, J., Ruder, H., \& Meszaros, P. 1996,ApJ, 467, 794

[19] Blum, S. \& Kraus, U. 2000, ApJ, 529, 968

[20] Giovannelli, F., Bernabei, S., Rossi, C., \& Sabau-Graziati, L. 2007, A\&A, 475, 651

[21] Foley, J., van Dam, A., Feiner, S., \& J., H. 1990, Computer Graphics: Principles and Practice (Addison-Wesley)

[22] Nollert, H.-P., Ruder, H., Herold, H., \& Kraus, U. 1989, A\&A, 208, 153

[23] Caballero, I. et al. 2008, in preparation 